http://www.ftsm.ukm.my/apjitm

Asia-Pacific Journal of Information Technology and Multimedia

Jurnal Teknologi Maklumat dan Multimedia Asia-Pasifik

Vol. 8 No. 1, June 2019: 17 - 26

e-ISSN: 2289-2192

\title{
HOMOGENEOUS GROUP PERFORMANCE AND STABILITY IN CHECKERS
}

\author{
THANATCHAI SIRIVICHAYAKUL \\ KRISTIAN SPOERER \\ HIROYUKI IIDA
}

\begin{abstract}
Experiments on groups of Checkers programs, playing by majority voting, were performed to investigate performance and stability. Homogeneous groups, copies of the same program, was used to perform these experiments instead of heterogeneous group that was more complicated by factors of different programs. Experiments were performed based on a search-depth of 5, 10 and 12 using the Samuel checkers program. Games of checkers were played between groups of size ranging from 1 up to 10 for each side. Experiment results suggest that group performance increases as a kind of logarithm function as the group size gradually increases for stronger player, and the performance slowly decreases in the case of a weaker player, stability seems to increase as the group size increases.
\end{abstract}

Keywords: Group performance, Homogeneous group, Stability, Checkers

\section{INTRODUCTION}

Studies on group benefit and performance have been conducted since 1898 (Triplett, 1898). In that study it was stated that collaboration of individuals, each completing the same task, resulted in the group outperforming each individual working alone. Many works indicated that group performance fluctuate on various aspect such as information sharing between group members (Hackman \& Morris, 1975), personality of members (Webber, 1974) and also group size (Thomas \& Fink, 1963). Group benefit, in the case of game research, under certain conditions also signifies that direction, however there are still some ambiguities and areas for improvement.

Since the birth of artificial intelligence (AI), games have been helping AI research progress. Games not only pose interesting and complex problems for AI to solve-e.g., playing a game well; they also offer a canvas for creativity and expression which is experienced by people or even machines. Thus, arguably, games are a rare domain where science meets art and interaction: these ingredients have made games a unique and favorite domain for the study of AI (Yannakakis \&Togelius, 2018). For example, good opening play in board games is quite important for developing superhuman AI as well as understanding the nature of opening book (Muangkasem, Iida \& Spoerer, 2013). Another example is the use of game elements in the educational context (Huynh \& Iida, 2017). Moreover, evolutionary changes of old games (Agarwal \& Iida) and entertainment assessment of video games (Anunpattana et al., 2018) are also intresting topics of game research using advanced AI technologies. A new trend of AI research using games is the notion of mixture-of-experts in the deep-learning context. It relates to a resarch idea known as group peformance in game playing on which we concentrate in this paper. 
Our present analysis relates to the well-known AI ensemble method (Russell \& Norvig, 2010). In ensemble methods, a group of individuals each make a proposal which is combined, for example using majority voting. The idea is that the probability of the majority making the same mistake is lower than the probability of an individual making the mistake, and therein lies the strength of the method.

\section{OBJECTIVE}

Many experiments and studies of Majority Voting indicate that, under certain conditions, the group Majority Voting performs better than the standalone components. Further studies and experiments are needed to understand those conditions, and may contribute to enhance the performance of Majority Voting.

The purpose of this research is to investigate Majority Voting. The principal aim is to find the relationship between performance and stability during Majority Voting, and factors which can further improve performance.

\section{METHODS}

\section{VARIABLES}

i. Group size: the number of player agents in a given side.

ii. Search Depth: Player's skill level.

iii. Player color: the side the player takes in the game; red and white. The player with the red pieces moves first.

\section{EXPERIMENT DESIGN}

Experiments on groups of Checkers programs were performed to test the effect of group size on performance and stability, with the 'simple majority voting' rule applied. Experiments were performed using a search-depth of 5, 10 and 12, with the Samuel checkers program ${ }^{1}$. The advantage of a player depends on search depth, as shown in 'An analysis of majority voting in homogeneous groups for checkers: Understanding group performance through unbalance' (Carvalho, Nguyen \& Iida, 2017), which shows the winning-rate of players in each depth and color. $W_{n}$ represents a group on white side made up of $n$ group size and $R_{m}$ represents a group on red side made up of $m$ group size.

First, the experiment was performed with the fixed search depth 10 because of suitability in strength and resource limit. Later, in consideration for relationship finding, experiments on search depth 5 and 12 were performed to compare and verify observations from the prior experiment. While focusing on selected search depth, experiments were performed with various changes in value of other variables.

\section{EVALUATION}

Observations were made from both players' perspective (white player and red player). A series of experiments were situationally prepared and the data was collected for statistical analysis. To evaluate the group performance, several statistics such as winning rate and average branching factor are compared and computed. The procedure of simple majority voting rule is as follows.

1. Compute $n$ candidate moves by searching with each of the $n$ programs in the group.

2. Sum the total count of each candidate move.

3. If there is a majority candidate, select it as the group move. 
4. If there is not a majority candidate, use the strongest member's proposed move to break the tie. In the case of a homogeneous group, the move of the first candidate is selected (Spoerer, Sirivichayakul \& Iida, 2013).

1,000 games were played between two checkers programs to find $W_{n}$ and $R_{m}$ (where 1 $\leq n, m \leq 8)$. The winning rate was calculated using the following equation (1).

$$
\text { Winning rate }=(\text { win }+0.5 * \text { draw }) / 1,000 \text {. }
$$

Average interval was used to smooth out short-term fluctuations in a series of data obtained from the experiments in order to more clearly recognize and analyze longer-term trends, cycles and stability. The average interval, or moving average, is sometimes referred to as a rolling average or a running average. A moving average is a series of numbers, each of which represents the average of an interval of specified number of previous periods. The 2 nd interval was used in this experiment.

The 2nd average interval can be calculated using the following equation (2).

$$
\text { avgInterval }_{n+1}=\left(\text { Winningrate }_{n}+\text { Winningrate }_{n+1}\right) / 2 \text {. }
$$

\section{RESULTS}

\section{EXPERIMENT ON SEARCH DEPTH 10}

Figure 1 shows the winning ratio of $W_{n}$ against $R_{m}$, with the overall average at around 0.6. From the viewpoint of performance, the winning ratio steadily swings around the average value and does not clearly show any specific sign of raising or lowering in value. However, from the viewpoint of stability we can see that with $n$ getting close to 1 , the difference between $n-1, n$ and $n+1$ becomes higher compared to when $n$ gets close to 8 .

Figure 2 shows the winning ratio of $W_{n}$ against $R_{m}$, with the overall average at around 0.77. After adding the factor of draw into the winning ratio, the results are similar to the results in Figure 1, the winning ratio swings steadily around average and with increasing $n$ the value gets closer to the average value.

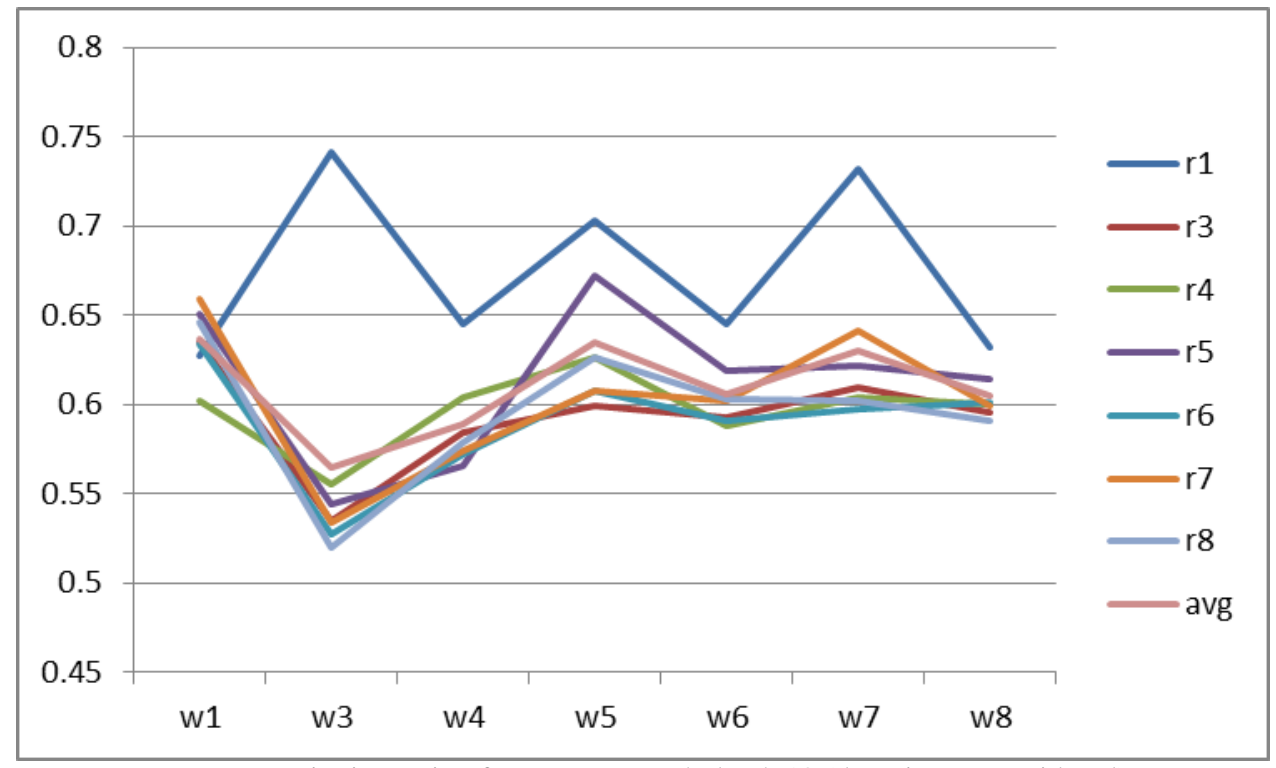

FIGURE 1. Winning ratio of groups at search depth 10 (draw is not considered) 


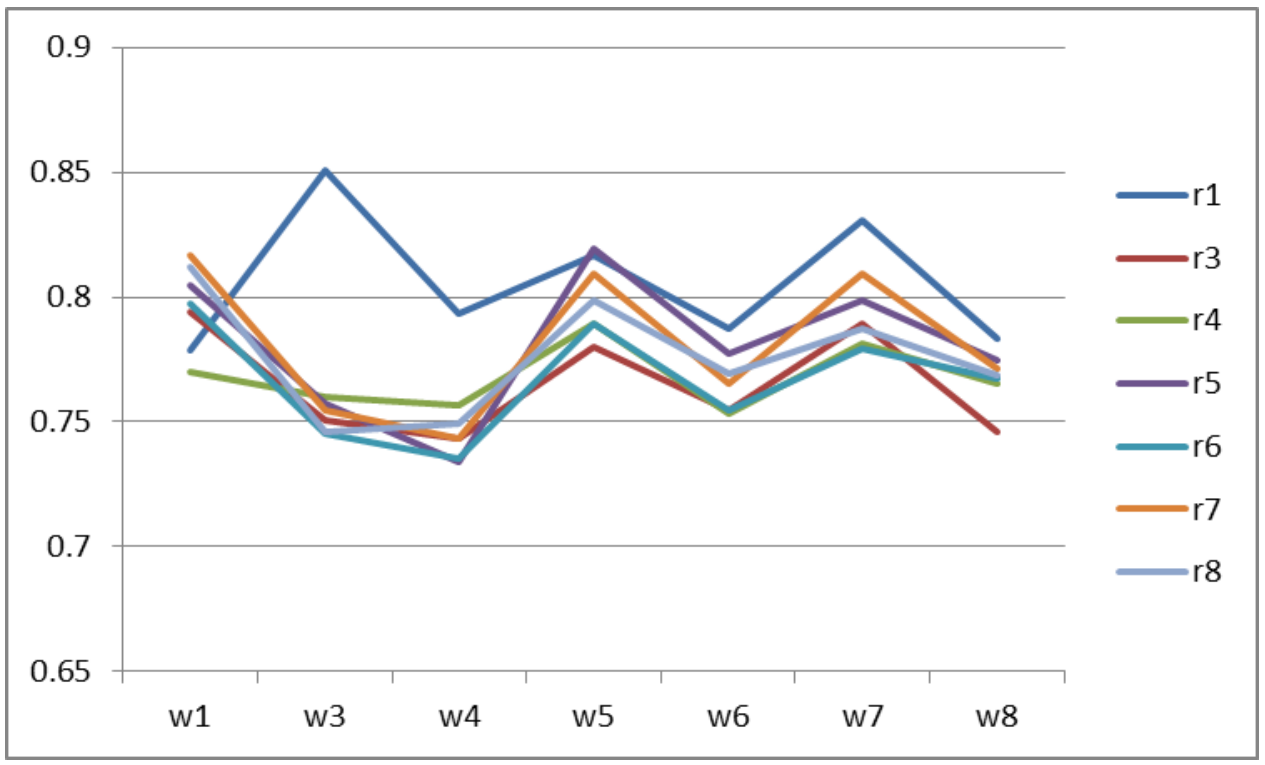

FIGURE 2. Winning ratio of groups at search depth 10 (draw is considered)

Figure 3 shows the average winning ratio of $W_{n}$ against $R_{m}$, with the overall average at around 0.78 . This figure shows an average value of Figure 2 for accuracy to analyse the overall results, and shows that the winning ratio swings steadily around the overall average. Additionally, with increasing the group size, the fluctuations decrease and the interval range between nodes becomes closer, which implies that the average value gradually stabilizes. The average interval also shows that there is a tendency for the winning ratio to increase as the group size increases.

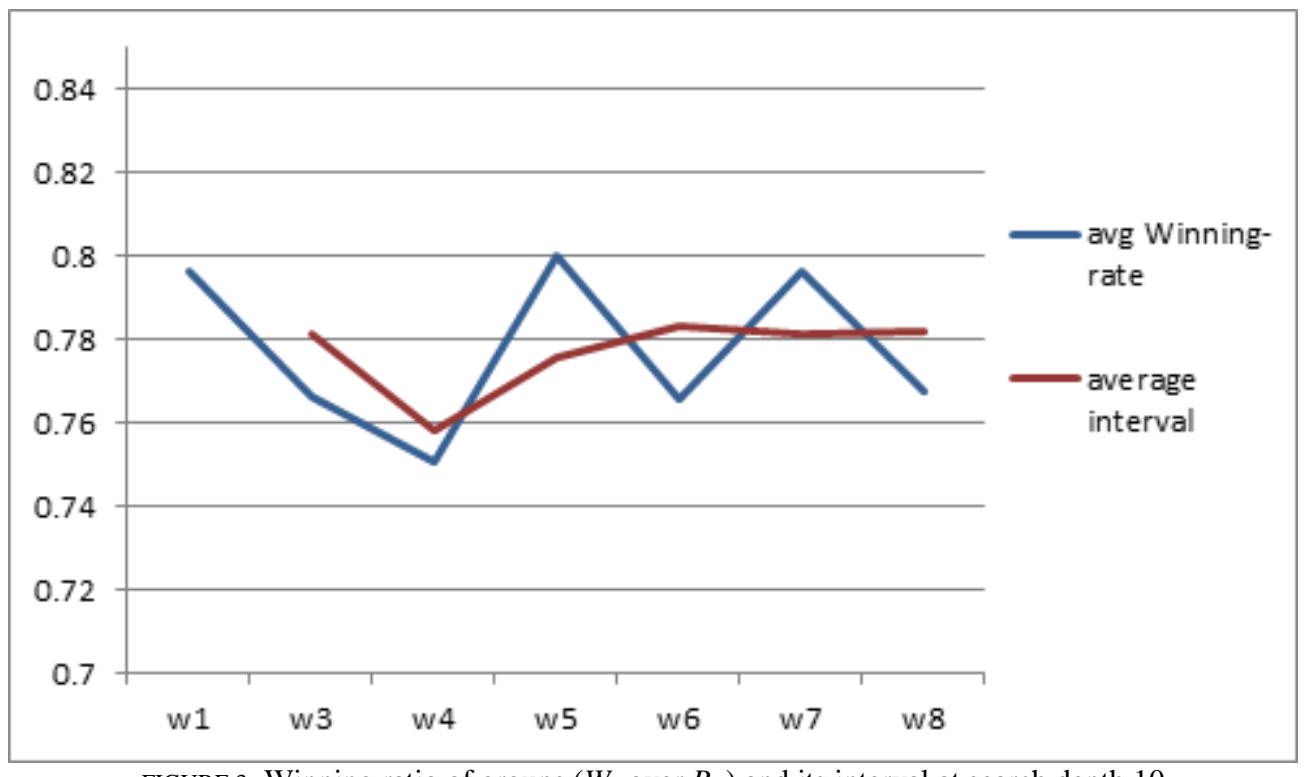

FIGURE 3. Winning ratio of groups $\left(W_{n}\right.$ over $\left.R_{m}\right)$ and its interval at search depth 10

Figure 4 shows the average winning ratio of groups $R_{m}$ against $W_{n}$. The graph shows that the winning ratio swings steadily around the overall average value. The interval range between nodes becomes closer as the group size increases, whereas the trend shows that the performance has a tendency to gradually decrease, which is similar to the results in Figure 3. 


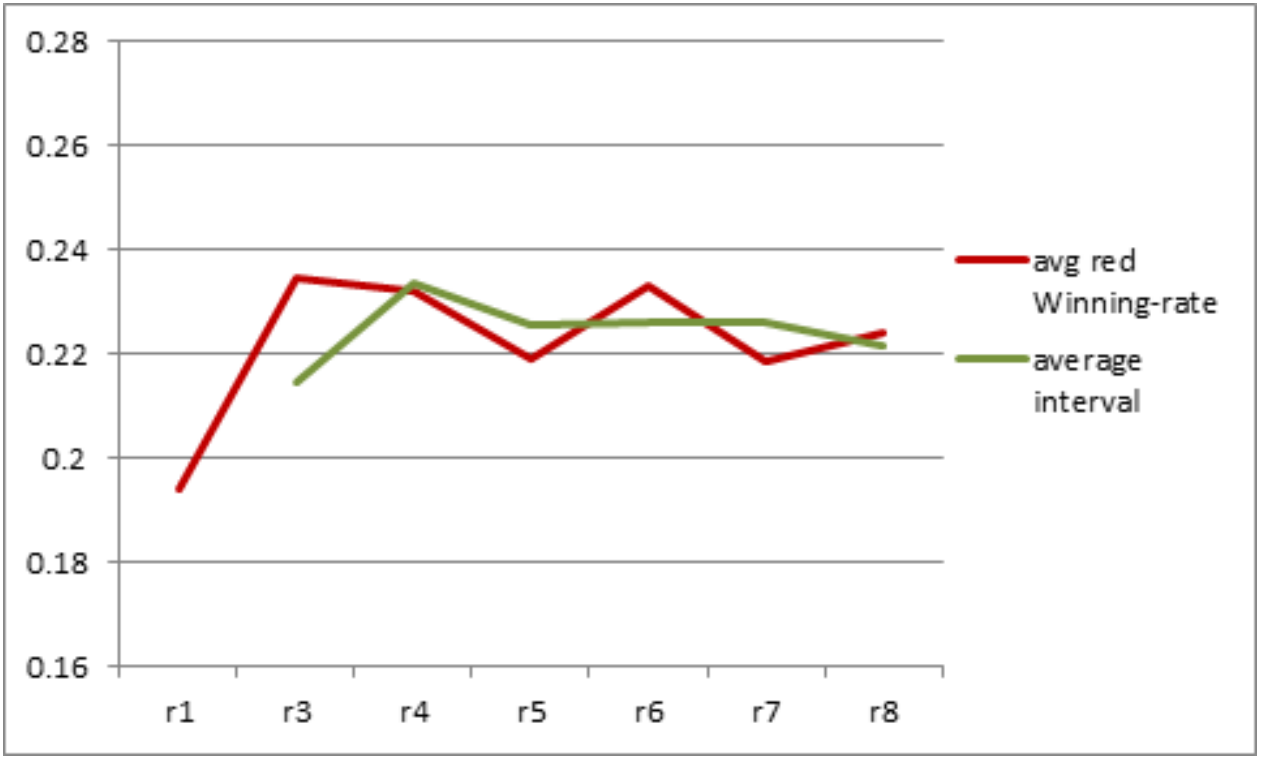

FIGURE 4 . Winning ratio of groups $\left(R_{m}\right.$ over $\left.W_{n}\right)$ and its interval at search depth 10

The results obtained from the experiments using a search depth of 10 can be summarised into 2 conjectures.

1. Conjecture 1: The winning ratio becomes more stable as the group size increases since fluctuations in the results decrease and the average value gradually stabilizes (Gorban, 2017).

2. Conjecture 2: For stronger players, the performance increases as the group size increases, whereas the performance decreases as the group size increases for weaker players.

\section{EXPERIMENT ON SEARCH DEPTH 5}

In order to confirm these hypotheses, experiments were performed with depth 5 and 12, with the group size starting from 1 up to 12. Figure 5 and Figure 6 show the result of experiments at search depth 5. In this level red is the stronger player in accordance with the results in related work (Carvalho, Nguyen \& Iida, 2017), but the analysis also resembles the experiment at search depth 10. Figure 5 shows the average winning ratio of $W_{n}$ against $R_{m}$ and the average interval, which decreases in performance as the group size increases. On the other hand, Figure 6 . shows the average winning ratio of $R_{m}$ against $W_{n}$ and the average interval, which increases in performance as the group size increases. Both figures show similar results, in fluctuations of the graph and the stability of the average value. 


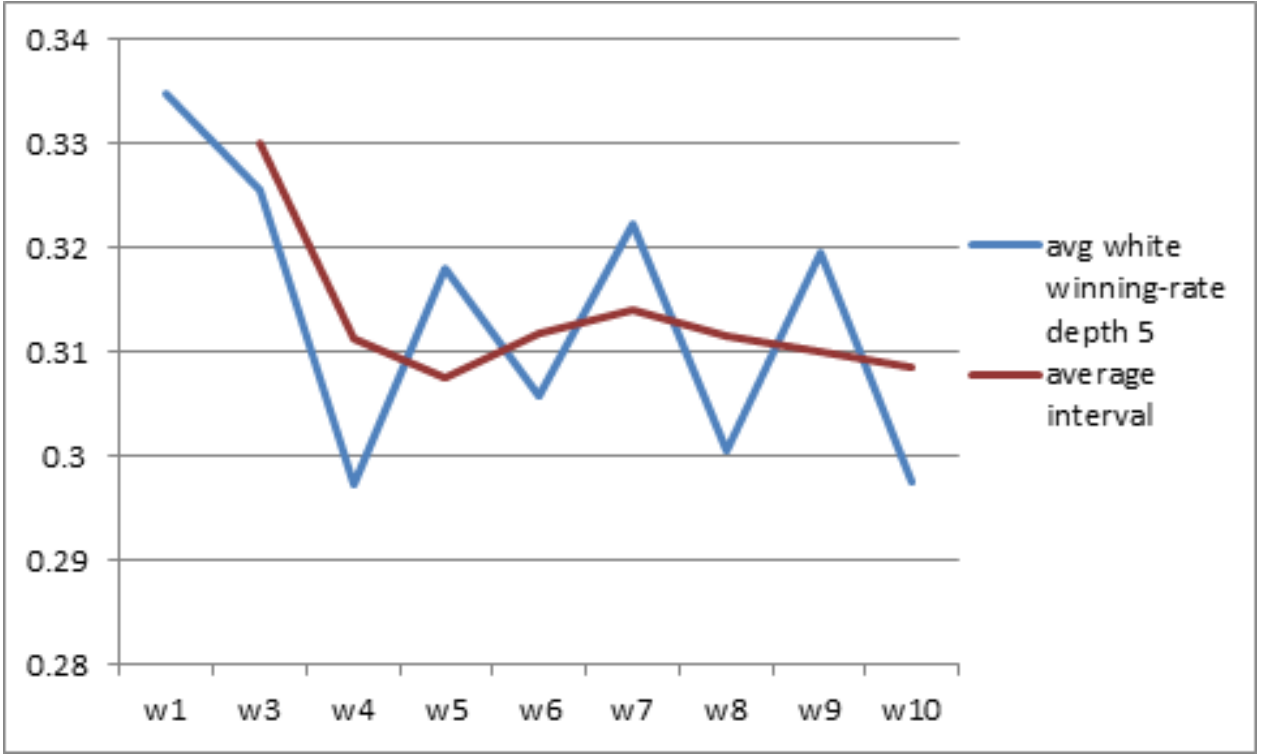

FIGURE 5. Winning ratio of groups ( $W_{n}$ over $\left.R_{m}\right)$ and its interval at search depth 5

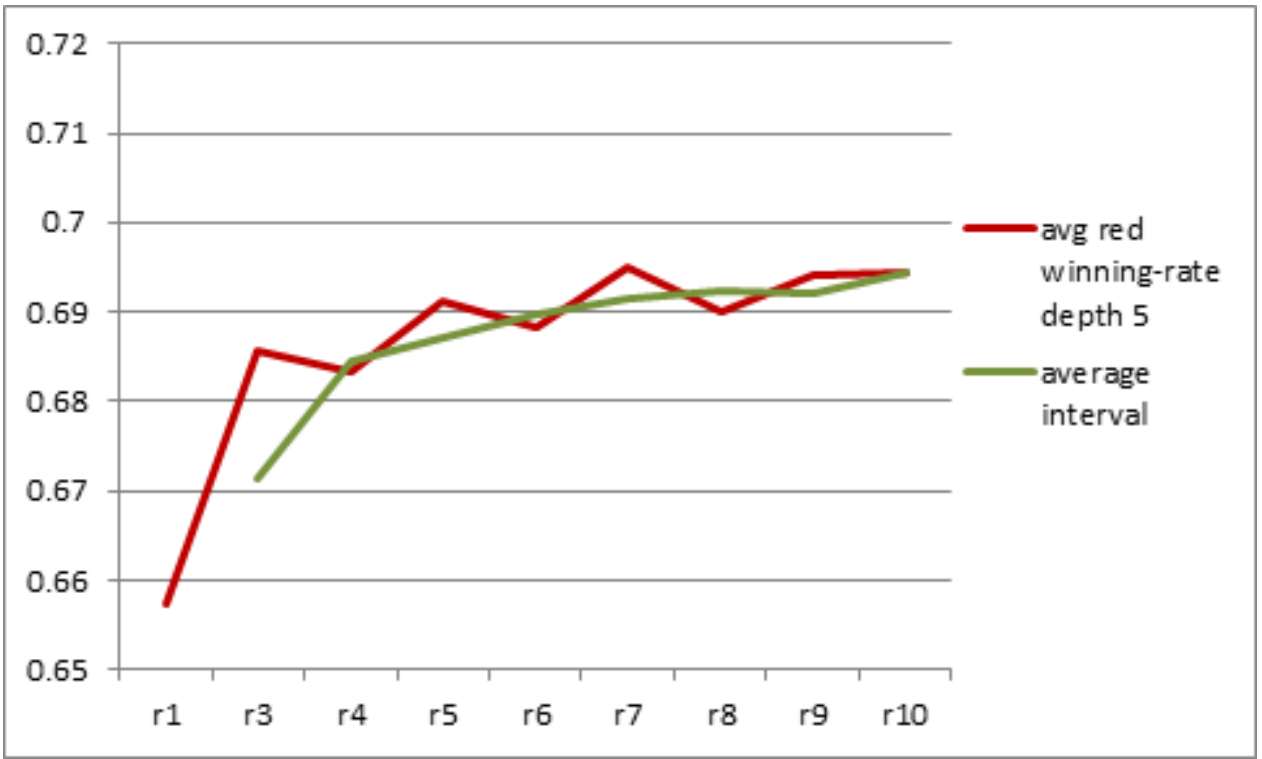

FIGURE 6. Winning ratio of groups $\left(R_{m}\right.$ over $\left.W_{n}\right)$ and its interval at search depth 5

Lastly, Figure 7 shows both average winning ratio of $W_{n}$ against $R_{m}$, the average winning ratio of $R_{m}$ against $W_{n}$ and the average interval at search depth 12 . The results show the exceptional performance by non-majority voting player (standalone program) which goes contrary to the presumption. However, ignoring the result from the non-majority voting player, the overall results performed by the majority voting group present a similar outcome to previous experiments. Group performance steadily improves as the group size increases for the stronger white, declining in group performance as the group size increases for the weaker red, and furthermore the average value stabilizes by decreasing in fluctuations. 


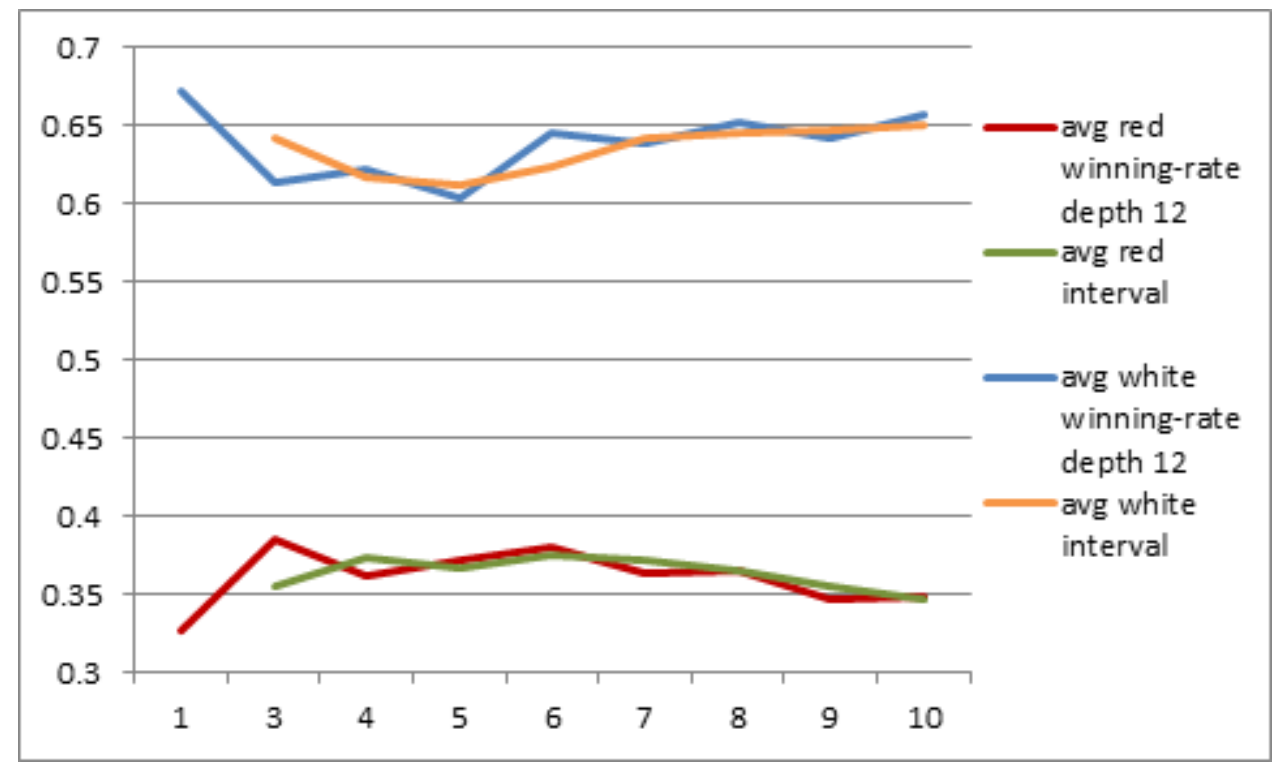

FIGURE 7. Winning ratio of groups $\left(W_{n}\right.$ over $\left.R_{m}\right),\left(R_{m}\right.$ over $\left.W_{n}\right)$ and its interval at search depth 12

\section{PROBABILITY ANALYSES}

The probability of the team picking moves which are a majority vote, $\operatorname{Pr} M$, is given by equation (3)

$$
\operatorname{Pr} M=\frac{M}{b^{n}},
$$

where $M$ is the number of all possible majority vote combinations and can be easily counted combinatorially. For a team size $1 \leq n \leq 8$, and using Checkers with an average branching factor $b$ of 3 , then $b^{n}$ is the total number of all move combinations, including majorities and ties, by the team.

The probability of the team picking moves are a tie, $\operatorname{Pr} T$, is easily given by equation (4).

$$
\operatorname{Pr} T=1-\operatorname{Pr} M \text {. }
$$


TABLE 1. Calculation of majority and tie probabilities

$$
n \quad M \quad b^{n} \quad \operatorname{Pr} M=\frac{M}{b^{n}} \quad \operatorname{Pr} T=1-\operatorname{Pr} M
$$

\begin{tabular}{c|cccc}
\hline 1 & 3 & 3 & 1 & 0 \\
\hline 2 & 3 & 9 & 0.333 & 0.666 \\
\hline 3 & 21 & 27 & 0.777 & 0.222 \\
\hline 4 & 63 & 81 & 0.777 & 0.222 \\
\hline 5 & 153 & 243 & 0.630 & 0.370 \\
\hline 6 & 579 & 729 & 0.794 & 0.206 \\
\hline 7 & 1767 & 2187 & 0.808 & 0.192 \\
\hline 8 & 4671 & 6561 & 0.712 & 0.288 \\
\hline
\end{tabular}

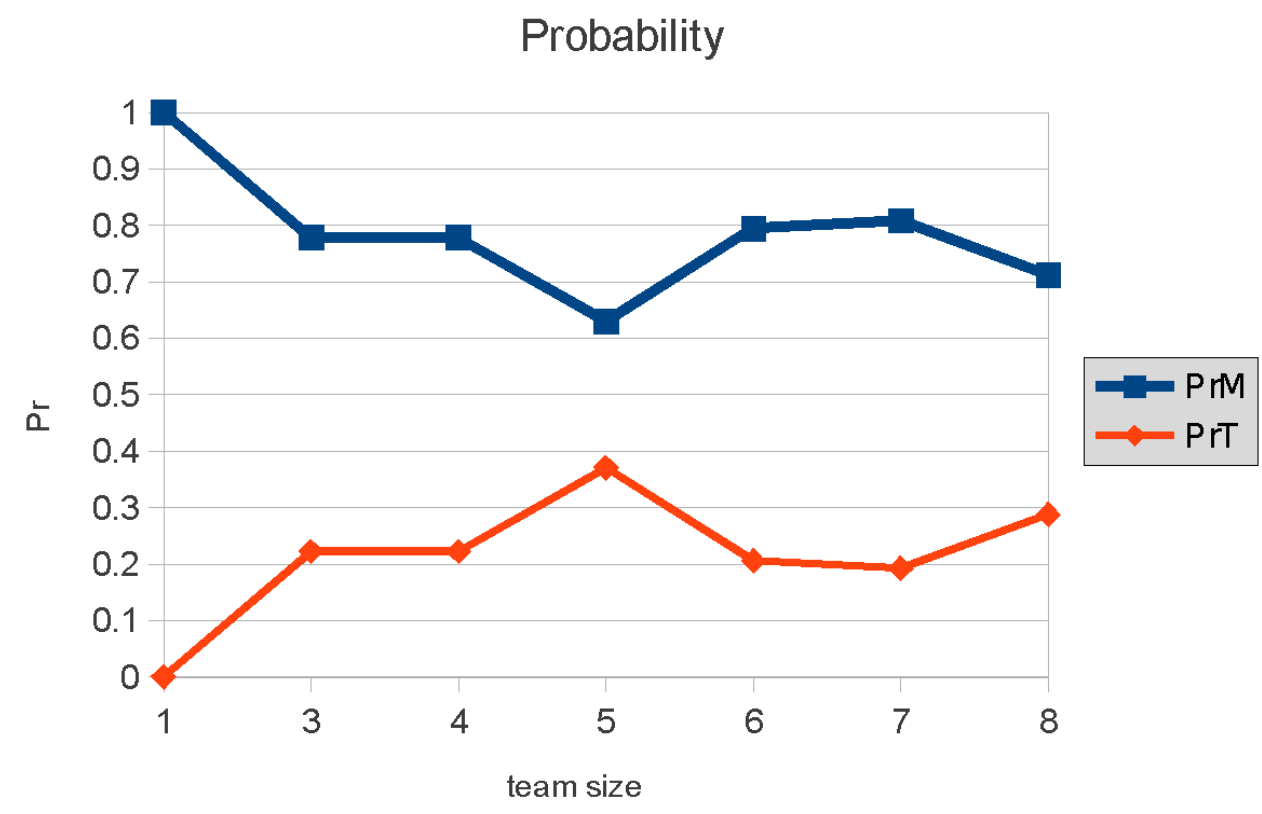

FIGURE 8. Probability of majority $\operatorname{Pr} M$ and tie $\operatorname{Pr} T$, for team size $n$.

Comparing $\operatorname{PrM}$ from Figure 8 and the winning ratio from Figure 2 shows some correlation, although team size 5 is inconsistent. In fact, for $\operatorname{Pr} T$ then team size 5 is consistent. The probability of the team picking moves which mean the leader's vote is played by the team, $\operatorname{Pr} L$, is given by equation (5)

$$
\operatorname{Pr} L=\frac{L}{b^{n}},
$$


where $L$ is the number of all possible situation combinations where the leader's move is picked and can be easily counted combinatorially by the following logic (6).

pickLeaderMove IFF isNotMajority OR (isMajority AND isLeaderInMajority)

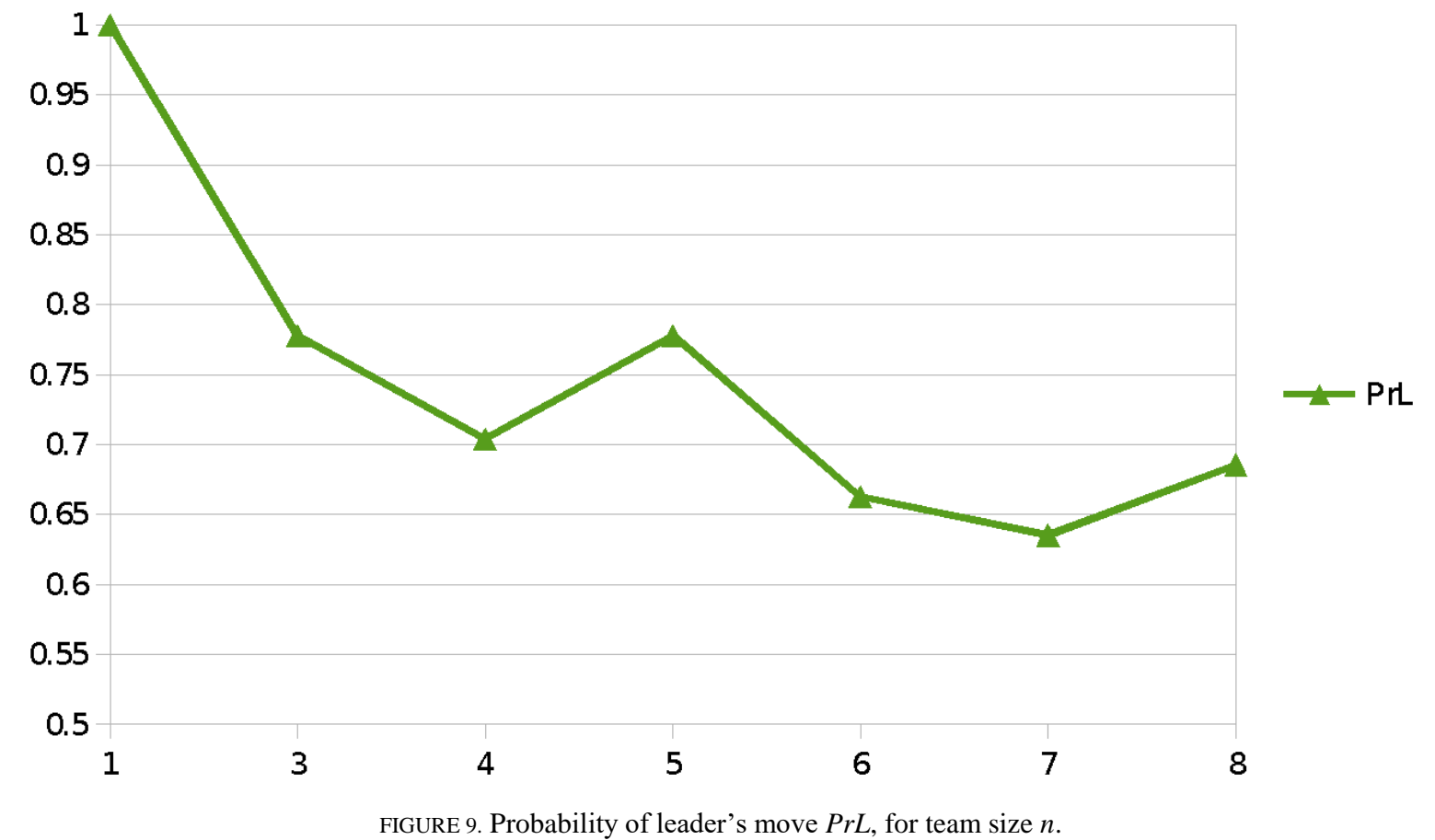

Comparing $\operatorname{PrL}$ from Figure 9 and the winning ratio from Figure 2, we see some correlation, although team size 7 is inconsistent.

\section{CONCLUSION}

In summary, experimental results, of majority voting in Checkers, suggest that group performance increases as a kind of logarithm function as the group size gradually increases for stronger player, and the performance slowly decreases in the case of a weaker player.

In addition, stability seems to increase as the group size increases. The result can be assumed that for higher $n$, the difference of the average interval of winning ratio between each node should be closer, which means the decreasing of fluctuations and an eventual stabilization of the average value.

We have also presented a probability analysis as a theory to explaining some of our present findings.

The results of this study can be concluded that with larger group size of a certain level players, majority voting would improve the group performance. Furthermore, that majority voting makes the homogeneous group more stable. It indicates a strong link between stability and quality in group performance.

\section{ACKNOWLEDGMENT}

This research is funded by a grant from the Japan Society for the Promotion of Science, within the framework of the Grant-in-Aid for Challenging Exploratory Research. 


\section{REFERENCES}

Agarwal, S., Iida, H. 2018. Analyzing Thousand Years Old Game Tigers and Goats is Still Alive, Asia-Pacific Journal of Information Technology and Multimedia, vol. 7, no. 2, pp. $1-11$.

Anunpattana, P., Khalid, M.N.A., Yusof, U.K., Iida, H. 2018. Analysis of Realm of Valor and ItsBusiness Model on PC and Mobile Platform Comparison, Asia-Pacific Journal of Information Technology and Multimedia, vol. 7, no. 2-2, pp. 1-11.

Carvalho, D.S., Nguyen, M.L., Iida, H. 2017. An analysis of majority voting in homogeneous groups for checkers: Understanding group performance through unbalance, Advances in Computer Games (M.H. Winands, H.J. van den Herik, and W.A. Kosters, eds.), (Cham), pp. 213-223, Springer International Publishing.

Hackman, J.R., Morris, C. G. 1975. Group tasks, group interaction process, and group performanceeffectiveness: A review and proposed integration, Advances in Experimental Social Psychology, vol. 8, pp. 45-99.

Huynh, D., Iida, H. 2017. An Analysis of Winning Streak's Effects in Language Course of "Duolingo", Asia-Pacific Journal of Information Technology and Multimedia, vol. 6, no. 2, pp. 23-29.

Muangkasem, A., Iida, H., Spoerer, K. 2013. Aspects of Opening Play, Asia-Pacific Journal of Information Technology and Multimedia, vol. 2, no. 1, pp. 49-56.

Spoerer, K., Sirivichayakul, T., Iida, H. 2013. Homogeneous group performance in chess, ProcediaTechnology, vol. 11, pp. 1272-1276. 4th International Conference on Electrical Engineering and Informatics, ICEEI 2013.

Russell, S., Norvig, P. 2010. Artificial Intelligence A Modern Approach. Third Edition, pp. 748.

Thomas, E.J., Fink, C.F. 1963. Effects of group size, Psychological Bulletin, vol. 60(4), pp. 371-384.

Triplett, N. 1898. The dynamogenic factors in pacemaking and competition, The American Journal of Psychology, vol. 9, no. 4, pp. 507-533.

Webber, R.A. 1974. The relation of group performance to the age of members of homogeneous groups, Academy of Management Journal, vol. 17, pp. 570-574.

Yannakakis, G. N., Togelius, J. 2018. Artificial Intelligence and Games. New York: Springer.

Thanatchai Sirivichayakul

Kristian Spoerer

Hiroyuki Iida

Japan Advance Institute of Science and Technology

1 Chome-1 Asahidai, Nomi, Ishikawa 923-1211, Japan

ice.thanatchai.s@gmail.com, iida@jaist.ac.jp

Received: 12 November 2018

Accepted: 15 April 2019

Published: 21 June 2019 